\title{
The Preovulatory Serum Estradiol Pattern in Natural IVF/ICSI Cycles
}

\author{
MILAN RELJIČl, ${ }^{1,2}$ and VELJKO VLAISAVLJEVIĆ ${ }^{\prime}$
}

Submitted: April 5, 1999

Accepted: June 8, 1999

Purpose: The aim of the study was to find whether inferences to the possible success of natural IVF/ICSI cycles could be drawn from the estradiol $\left(E_{2}\right)$ pattern.

Methods: Sixty-eight women who underwent oocyte recovery in 98 natural cycles were recruited for the study. Daily serum $E_{2}$ was measured in the preovulatory phase $(-3$ to +2 day). The $E_{2}$ pattern was compared among four groups: Group $A$, unsuccessful egg retrieval; Group $B$, no fertilization; Group $C$, no implantation; and Group D, implantation.

Results: There was no difference in mean $E_{2}$ levels between groups. Only the ratio of $E_{2}$ on day $+I / E_{2}$ on day 0 was significantly lower in conception cycles in comparison with nonconception cycles. In cycles with a decreased $E_{2}$ level on day $+l$, only the implantation rate was significantlly higher in comparison with cycles with an increasing $E_{2}$ level. Conclusions: From the $E_{2}$ pattern it is possible to make inferences about the likelihood of implantation but not the fertilization or oocyte recovery success.

KEY WORDS: estradiol pattern; implantation; natural cycles; in vitro fertilization.

\section{INTRODUCTION}

The first successful pregnancy resulting from in vitro fertilization (IVF) occurred during an unstimulated normal menstrual cycle $(1,2)$. Shortly afterward, however, most IVF programs began using controlled ovarian hyperstimulation $(\mathrm{COH})$ to achieve the development of several preovulatory follicles in an effort to increase the number of available embryos and consequently improve pregnancy success rates (3). Since then, improvements

\footnotetext{
' Unit for Human Reproduction and Endocrinology, Department of Gynecology and Perinatology, Maribor Teaching Hospital, Maribor, Slovenia.

${ }^{2}$ To whom correspondence should be addressed at Department of Gynecology and Perinatology, Maribor Teaching Hospital, Ljubljanska 5, SI-2000 Maribor, Slovenia.
}

occurred in oocyte culture technique, sperm preparation, and oocyte retrieval. Those improvements created a renewed interest in using spontaneous cycles in IVF (4).

In most recent reports of IVF in spontaneus cycles, human chorionic gonadotropin (hCG) has been administered to replace (and precede) the natural luteinizing hormone $(\mathrm{LH})$ surge to facilitate the timing of oocyte recovery and planning of work schedules (5). The designation of this type of cycle has been natural or unstimulated IVF (6). Those cycles are successful and have several advantages compared with stimulated cycles. Despite this, natural cycles are not widely used in IVF programs (7). The main reason is the high failure rate at each step in the process and the unacceptably low delivery rate per egg recovery attempt, compared with stimulated cycles (8). To improve the pregnancy rate with natural cycles, it is important to select the patient according to age, infertility factors, basal LH level, etc. $(6,9-11)$. Besides, we also know that the fecundibility of all cycles is not equal in the same woman. So it is also important to identify the factors that predict the outcome of a certain cycle.

The aim of our study was to establish whether it was possible to make inferences about the success of natural IVF intracytoplasmic sperm injection (ICSI) cycles from the periovulatory serum estradiol $\left(E_{2}\right)$ pattern.

\section{MATERIALS AND METHODS}

Sixty-eight women who underwent oocyte recovery in 98 natural cycles were recruited for this study. Their age range was $25-41$ years (mean $=33$ years, $S D=$ \pm 4.4 years). They were chosen from among those couples in which a stimulated IVF/ICSI procedure was anticipated. The indications for IVF/ICSI procedure were only female infertility in $45(45.9 \%)$, only male infertility in $17(17.3 \%)$, female and male infertility in 
$11(11.2 \%)$, and unexplained infertility in $25(25.5 \%)$ cycles. In female infertility tubal infertility prevailed (54, or $55.1 \%$ ); in male infertility, oligozoospermia $(12$, or $12.2 \%)$. In $24(24.5 \%)$ women with tubal infertility, hydrosalpinx was established previously. A pelvic ultrasound examination was performed on day 2 of the menstrual cycle to evaluate the ovaries for the presence of cysts and on the same day serum follicle stimulating hormone (FSH) and $\mathrm{E}_{2}$ were measured to confirm that the cycle has just begun. The cycle was canceled if the baseline $\mathrm{E}_{2}$ was $>0.3 \mathrm{n} M$. Transvaginal ultrasonography was performed and the serum $E_{2}$ concentration was measured every 2 days from day 7 onward, and every day when the follicular diameter was $>12 \mathrm{~mm}$ and the serum $\mathrm{E}_{2}$ concentration $>0.3$ $\mathrm{n} M$. Cycles with more than one growing follicle $>12$ $\mathrm{mm}$ were not included in the study.

hCG (5000 IU) was administered at $2100 \mathrm{hr}$ when the average follicular diameter was $>15 \mathrm{~mm}$ and the serum $\mathrm{E}_{2}>0.5 \mathrm{nM}$. Blood was drawn for $\mathrm{E}_{2}$ estimation 17-18 hr after hCG application and just before oocyte recovery. At 36-37 hr after hCG injection, follicular puncture was carried out in patients with negative $\mathbf{L H}$ before hCG application. The oocytes were cultivated and inseminated, and the embryos transferred as described previously (12). The luteal phase was supported with hCG (1000 IU) on the day of embryo transfer and 4 and 9 days later.

Serological documentation of pregnancy was scheduled 16 days after embryo transfer. A pregnancy was confirmed only if there was ultrasonic evidence of a gestation sac.

The $E_{2}$ pattern was compared among four groups of cycles: Group A, unsuccessful egg retrieval; Group $\mathrm{B}$, no fertilization; Group $\mathrm{C}$, no implantation; and Group D, implantation. $E_{2}$ levels on a certain day and also the ratio of $E_{2}$ levels between 2 consequent days were compared using the one-way ANOVA. If a statistically significant association was found between the $\mathrm{E}_{2}$ ratio and the cycle outcome, the cycles were divided into two groups according to the ratio value and the difference between two groups was evaluated with Fischer's exact and Mann-Whitney $U$ tests. The data were processed with the statistical program by Statistica. Statistical significance was set at $P<0.05$. The study was approved by the Ethics Committee at the Ministry of Health of the Republic of Slovenia.

\section{RESULTS}

The mean FSH level observed on day 2 of the cycle was $7.32 \pm 3.12 \mathrm{IE}$. The mean follicular diameter on the day of hCG injection was $16.4 \pm 1.01 \mathrm{~mm}$. In a total of 98 follicular punctures, 66 oocytes were recovered (recovery rate $=67.3$ ). In $11(16.7 \%$ ) cycles ICSI was performed; and in $55(83.3 \%)$, IVF. The overall fertilization rate was $66.7 \%(43 / 66)$. Ten clinical pregnancies resulted, representing an implantation rate of $23.3 \%$ (10/43) and a pregnancy rate of $10.2 \%$ (10/98) per attempted egg recovery. According to these results Group A consisted of 32, Group B of 23, Group C of 33, and Group D of 10 cycles. No differences were established between the groups as regards indications for the IVF/ICSI procedure, the percentage of ICSI procedures, the woman's age, the percentage of women with hydrosalpinx, the FSH level on day 2 of the menstrual cycle, and the mean follicular diameter on the day of hCG injection.

The pattern of serum $E_{2}$ in the periovulatory phase in all four groups is shown in Fig. 1. The mean $E_{2}$ level rose daily from day -3 up to hCG administration (day 0 ) and is similar in all four groups. In nonconception cycles (Groups A, B, and C), an increase in mean $E_{2}$ levels was observed from day 0 onward, peaking on day +1 and then decreasing toward day +2 . In conception cycles (Group D), a continual decrease was observed from day 0 to day +2 . However, there was no statistically significant difference in mean $E_{2}$ levels between groups on a certain day. Only the $E_{2}$ on day $+1 / \mathrm{E}_{2}$ on day 0 ratio was statistically significantly lower in conception cycles in comparison with nonconception cycles (Table I). If we divided the cycles into two groups according to different values of this ratio, we would find that only the implantation rate was different. There was no implantation if the $E_{2}$ level on day +1 increased more than $10 \%$ from the previous day. In cycles with the same or a decreased $E_{2}$ level on day +1 , the implantation rate was statistically significantly higher in comparison with the implantation rate in cycles with an $\mathrm{E}_{2}$ level increasing up to $10 \%$ on day +1 (Table II). No differences were established between the groups as regards indications for the IVF/ ICSI procedure, the percentage of ICSI procedures, the woman's age, the percentage of women with hydrosalpinx, the FSH level on day 2 of the menstrual cycle, and the mean follicular diameter on the day of hCG injection and $E_{2}$ levels before and on day 0 .

\section{DISCUSSION}

Serum $E_{2}$ measurement is important in monitoring natural cycles and, according to some opinions, also for predicting cycle outcome. In reviewing the characteristics of successful and unsuccessful cycles in natu- 


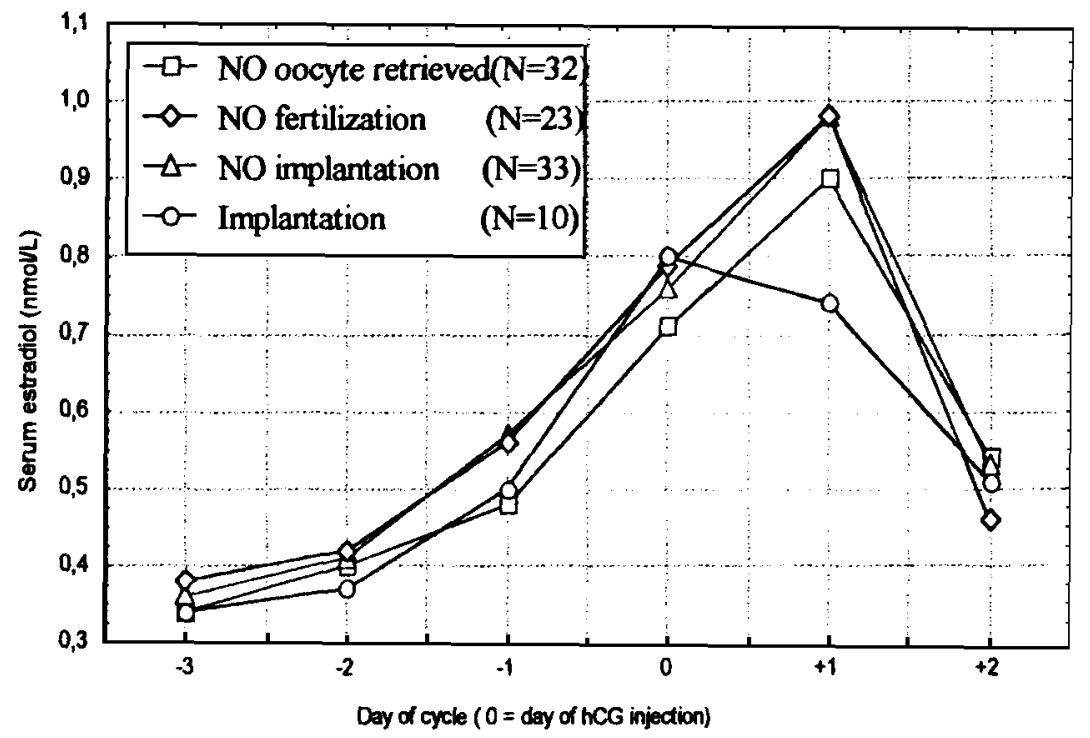

Fig. 1. Preovulatory serum estradiol pattern in natural IVF/ICSI cycles.

Table I. $E_{2}$ Levels on Days 0 and +1 and $E_{2}$ on Day $0 / E_{2}$ on Day +1 Ratio in Four Groups, Analyzed by One-Way ANOVA

\begin{tabular}{|c|c|c|c|}
\hline \multirow[b]{2}{*}{ Group } & \multicolumn{2}{|c|}{$\mathrm{E}_{2}($ mean $\pm \mathrm{SD} \mathrm{n} M)$} & \multirow{2}{*}{$\begin{array}{c}E_{2} \text { on day } 0 / E_{2} \text { on day }+1 \\
(\text { mean } \pm S D)\end{array}$} \\
\hline & Day 0 & Day + 1 & \\
\hline No oocyte retrieved (A) & $0.709 \pm 0.202$ & $0.901 \pm 0.254$ & $1.315 \pm 0.354$ \\
\hline No fertilization (B) & $0.760 \pm 0.238$ & $0.979 \pm 0.418$ & $1.291 \pm 0.409$ \\
\hline No implantation (C) & $0.797 \pm 0.313$ & $0.980 \pm 0.320$ & $1.286 \pm 0.364$ \\
\hline Implantation (D) & $0.798 \pm 0.177$ & $0.740 \pm 0.221$ & $0.914 \pm 0.127^{*}$ \\
\hline All groups & $0.760 \pm 0.250$ & $0.930 \pm 0.323$ & $1.259 \pm 0.370$ \\
\hline
\end{tabular}

* Statistically significant $(F=3.51, P=0.0182)$.

ral IVF programs, Paulson noted that cycles resulting in pregnancies tended to have a higher $\mathrm{E}_{2}$ level on the day of hCG application than cycles which failed (6). However, this is of questionable practical value, because waiting for the attainment of a higher $\mathrm{E}_{2}$ level may result in the occurrence of an endogenous $\mathrm{LH}$ surge and a potential loss of the cycle (6). On the other side, there is not always a correlation among oocyte maturity, cycle fecundibility, and $E_{2}$ level on the day of $\mathrm{hCG}$ application. So the $\mathrm{E}_{2}$ level on a certain day

Table II. Outcome of Natural IVF/ICSI Cycles According to Different $E_{2}$ on Day $0 / E_{2}$ on Day +1 Ratios

\begin{tabular}{llllllll}
\hline & \multicolumn{5}{c}{$\mathrm{E}_{2}$ on day $0 / \mathrm{E}_{2}$ on day +1} \\
\cline { 2 - 6 } & $\leq 0.9$ & $>0.9$ & $\leq 1$ & $>1$ & $\leq 1.1$ & $>1.1$ & Total \\
\hline No. of cycles & 14 & 84 & 26 & 72 & 39 & 59 & 98 \\
Oocyte recovery rate & 78.5 & 65.5 & 73.4 & 65.3 & 71.8 & 64.5 & 67.3 \\
Fertilization rate & 72.7 & 65.5 & 78.9 & 61.7 & 71.4 & 63.2 & 66.7 \\
Implantation rate & $71.4 *$ & 13.9 & $50.0 *$ & 10.3 & $52.6 *$ & 0.0 & 23.3 \\
\hline
\end{tabular}

* Statistically significant $(P<0.01)$. in the cycle is not convenient for predicting the cycle outcome. Our own results confirm this, as there was no statistically significant difference between groups with different cycle outcomes in mean $E_{2}$ levels on a certain day in the periovulatory phase.

But we found that it was possible to predict the outcome of IVF/ICSI cycles on the basis of the $E_{2}$ pattern. It is remarkable that we established a difference in $E_{2}$ pattern only in dependence on implantation, and not on fertilization or oocyte recovery success. Although other authors also do not find a connection between the $\mathrm{E}_{2}$ pattern and fertilization (13), we admit the possibility that our results were influenced by the choice of indications for an IVF/ICSI cycle. Our study also included couples treated for male infertility, including ICSI cycles. Although we would expect the possibility of successful oocyte recovery to depend on the $E_{2}$ pattern and $E_{2}$ levels on the day of hCG application, we did not make this observation. Daya et al. reached similar conclusions. They believe that the success of follicle puncture depends more on the 
choice of the needle and the technique than the $E_{2}$ levels (9). As regards the possibility of predicting implantation, our findings are quite different. We established a difference in $E_{2}$ pattern between conception and nonconception cycles immediately after hCG application. In all conceptional cycles the $\mathrm{E}_{2}$ level did not increase for more than $10 \%$ on the day following hCG application; in the majority it remained the same or dropped. The $\mathrm{E}_{2}$ pattern in conception cycles in our study resembles the dynamics of $E_{2}$ in a normal menstrual cycle, in which, at about the time of the preovulatory $\mathrm{LH}$ surge, $\mathrm{E}_{2}$ levels do not rise and decline within $24 \mathrm{hr}$ after the onset of the LH surge (14). However, different $\mathrm{E}_{2}$ patterns were observed by Jones et al. (15) and Laufer et al. (16) in conception $\mathrm{COH}$ cycles. They found the highest pregnancy rate among patients whose $E_{2}$ levels continued to rise on the day after hCG administration in COH cycles $(15,16)$. Dor et al. also found that almost all $(96 \%)$ women in their study with conceptional cycles had rising or plateauing $\mathrm{E}_{2}$ levels on the day after hCG, whereas $37.5 \%$ of the women had decreasing $E_{2}$ levels (13).

Some observations in $\mathrm{COH}$ cycles and normal menstrual cycles as well as our own observations in natural cycles show that $\mathrm{E}_{2}$ dynamics after hCG application may depend on follicular maturity. Dor et al. supposed that a decrease in $E_{2}$ levels in nonconceptional stimulated cycles was associated with follicular athresia and unruptured hemorrhagic follicles (13). The difference in $E_{2}$ patterns in stimulated and natural conception cycles can also be explained by the influence of follicular maturity on $E_{2}$ secretion. Numerous follicles grow in stimulated cycles, and since this growth is not necessarily synchronic, follicular maturity and $E_{2}$ pattern are different. In a normal menstrual cycle it is assumed that the endogenous $\mathrm{LH}$ release is synchronized with optimal follicular and oocyte maturity, so serum $E_{2}$ levels decrease after the initial rise in luteinizing hormone level due to a decrease in androgen production by theca cells and a similar fall in the aromatase activity of granulosa cells (17). These findings indicate that in our study, in those natural cycles in which there was no estradiol increase on day +1 , the hCG application was performed in time, follicular maturity was optimal, and the possibility of conception was greater. In cycles with an $E_{2}$ increase it was too early, thus decreasing the possibility of conception. According to our observations, the $E_{2}$ levels on the day of hCG application do not influence further $E_{2}$ dynamics or reflect follicular maturity since no differences were found between average $E_{2}$ levels with regard to different $E_{2}$ patterns. This is understandable since, according to experience in the normal menstrual cycle, accurate determination of follicle maturity is very difficult to recognize on the $E_{2}$ level if we know that the observed ranges for serum $\mathrm{E}_{2}$ were $0.451-3.071 \mathrm{p} M$ at the onset of the spontaneus LH surge (18).

On the basis of the importance of timely application, we might conclude that the pregnancy rate would be higher if the endogenous LH surge is used to determine the time of oocyte recovery, which is not the case. The reason lies in the advantage of hCG administration allowing much accurate timing of oocyte retrieval, which is probably of importance since the duration of oocyte maturation in vivo before aspiration appears to influence the chance of pregnancy (18). If it is possible to deduce the favorable time of hCG application retrogradely, the pregnancy rate per oocyte recovery can be improved by choosing cycles with a greater possibility of implantation, on the basis of $E_{2}$ levels on the day of hCG application and the day after, and performing the IVF/ICSI procedure in these only. We should realize that in view of the small number of cycles, the conclusions regarding predictability are preliminary. There is also the possibility that we may not have included all the factors bearing an influence on the $E_{2}$ pattern as well as on the implantation of embryos. Therefore, before clinical application, these results should be confirmed in a larger number of cycles, taking the remaining factors affecting the outcome of natural IVF/ICSI cycles into consideration.

\section{ACKNOWLEDGMENTS}

The authors wish to thank Marijana Gajšek-Marchetti, translator, from the Medical Research Department, for her contribution in preparing the manuscript. This study was sponsored by Ministry of Science and Technology of the Republic of Slovenia Grant No. J3-8764.

\section{REFERENCES}

1. Edwards RG, Steptoe PC, Purdy JM: Establishing full-term human pregnancies using cleaving embryos grown in vitro. $\mathrm{Br}$ J Obstet Gynecol 1980;87:737-756

2. Stepoe PC, Edvards RG: Birth after the reimplantation of a human embryo. Lancet 1978;2:366

3. Paulson RJ, Sauer MV, Lobo RA: In vitro fertilization in unstimulated cycles: A new application. Fertil Steril 1989;51:1056-1060

4. Garcia J: Return to the natural cycle for in vitro fertilization/ Alleluia, alleluia!/. J Vitro Fert Embryo Transfer 1989;6:67-68 
5. Fahy UM, Cahill DJ, Wardle PG, Hull MGR: In-vitro fertilization in completely natural cycles. Hum Reprod 1995;10:572-575

6. Paulson RJ: Natural cycle in vitro fertilization. Infertil Reprod Med Clin North Am 1993;4:653-665

7. Vlaisavljević V, Gavrič V, Kovačič B: In vitro fertilization in natural cycles: Maribor experience. In 9 th World Congress on In Vitro Fertilization and Assisted Reproduction, 3-7 April 1995, Vienna (Austria), A Aburumieh, E Bernat, G Dohr, W Feichtinger, F Fischl, J Huber, E Müller, S Szalay, W Urdl, H Zech (eds). Bologna, Monduzzi Editore, 1995, pp 573-575

8. Lenton EA, Woodward B: Controversies in assisted reproduction. Natural versus stimulated cycles in IVF: Is there a role for IVF in natural cycle? J Assist Reprod Genet 1993;10:406-408

9. Daya S, Gunby J, Hughes EG, Collins JA, Sagle MA, YoungLai EV: Natural cycles for in vitro fertilization: Cost-effectiveness analysis and factors influencing outcome. Hum Reprod 1995;10:1719-1724

10. Seibel MM, Kearnan M, Kiessling A: Parameters that predict success for natural cycle in vitro fertilization-embryo transfer. Fertil Steril 1996;63:1251-1254

11. Monks NJ, Turner K, Hooper MAK, Kumar A, Verma S, Lenton EA: Development of embryos from natural cycle in-vitro fertilization: Impact of medium type and female infertility factors. Hum Reprod 1993;8:266-271

12. Vlaisavljević V, Kovačič B, Gavrić V. In vitro fertilization program based on programmed cycles monitored by ultrasound only. Int J Gynecol Obstet 1992;39:227-231
13. Dor J, Rudak E, Mashiach S, Nebel L, Serr DM, Goldmqan B: Periovulatory $17 \beta$-estradiol changes and embryo morphologic features in conception and nonconception cycles after human in vitro fertilization. Fertil Steril 1986;45:63-68

14. Djahanbakhch O, McNeilly AS, Warner PM, Swanston IA, Baird DT: Changes in plasma levels of prolactin, in relation to those of FSH, oestradiol, androstenedione and progesterone around the preovulatory surge of LH in women. Clin Endocrinol 1984;20:463-472

15. Jones HW, Acosta A, Andrews MC, Garcia JE, Jones GS, Mantzavinos T, McDowell J, Sandow B, Veeck L, Whibley T, Wilkes C, Wright G: The importance of follicular phase to success and failure in in vitro fertilization. Fertil Steril 1983:40:317

16. Laufer N, DeCherney AH, Tarlatzis BC, Naftolin F: The association between preovulatory serum $17 \beta$-estradiol pattern and conception in human menopausal gonadotropin-human chorionic gonadotropin stimulation. Fertil Steril 1986;46:73-76

17. Hiller SG, Reichert LE, Van Hall EV: Control of preovulatory follicular estrogen biosynthesis in the human ovary. J Clin Endocrinol Metab 1981;52:847

18. Cahill DJ, Wardle PG, Harlow CR, Hull MGR: Onset of the preovulatory luteinizing hormone surge: Diurnal timing and critical follicular prerequisites. Fertil Steril 1998;70:5659

19. Stepoe PC, Edvards RG, Purdy JM: Clinical aspects of pregnancies established with cleaving embryos grown in vitro. $\mathrm{Br} J$ Obstet Gynaecol 1980;87:757-786 